\title{
Transparent Conducting Contacts Based on Zinc Oxide Substitutionally Doped with Gallium
}

Christopher W. Gorrie*, Matthew Reese, John D. Perkins, Jeff L. Alleman, Matthew S. Dabney, Bobby To, David S. Ginley, Joseph J. Berry

National Center for Photovoltaics, National Renewable Energy Laboratory, 1617 Cole Blvd., Golden Colorado 80401 USA

*Department of Materials Science and Engineering, University of Florida, Gainesville, Florida 32611 


\section{Motivation}

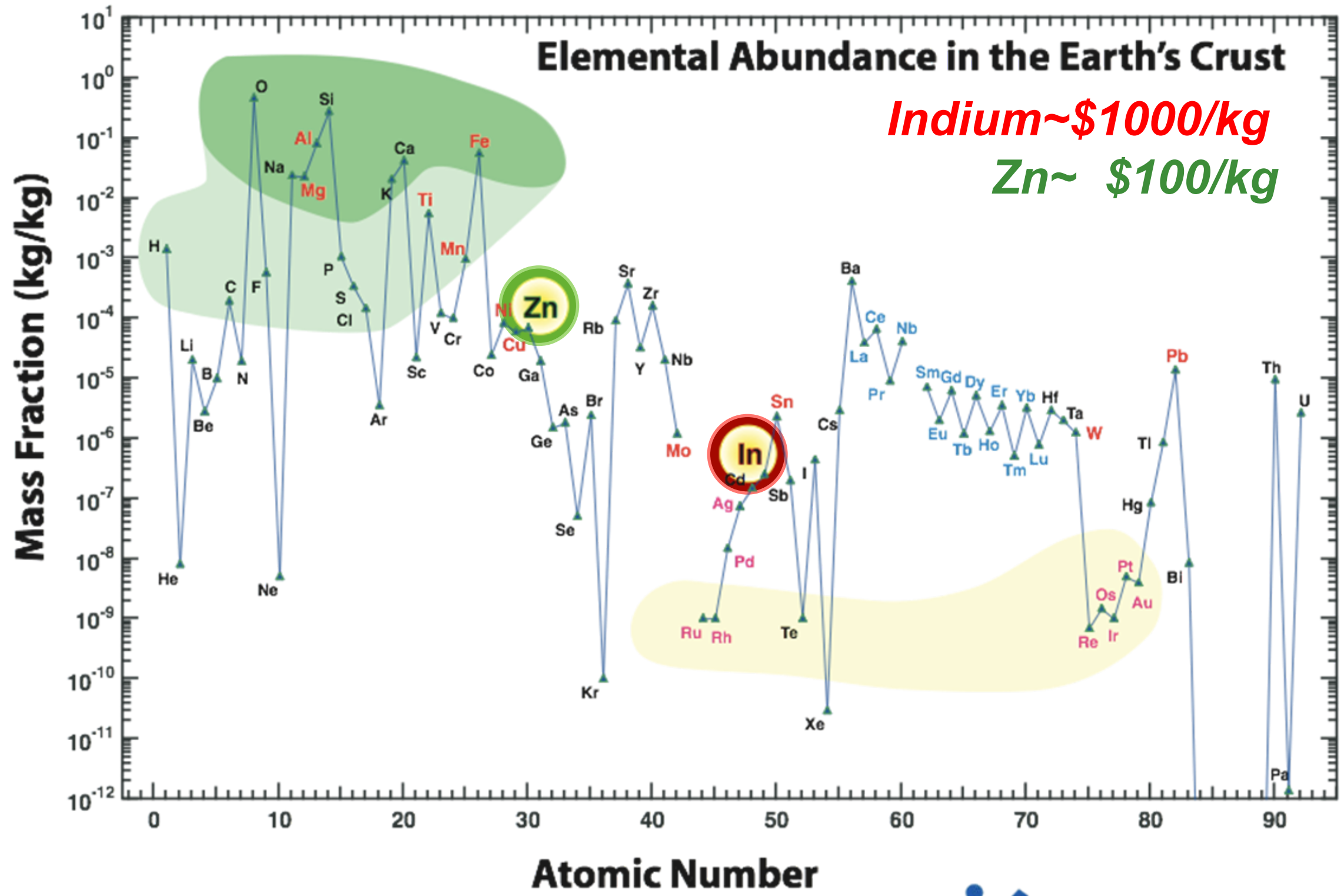

NREL is operated by Midwest Research Institute - Battelle 


\section{ZnO}

- Band gap Engineering

- Cd:ZnO

- Decreased gap

- Mg:ZnO

- Increase gap

- Doping

- Al:ZnO

- Conventional

- Ga:Zno

- Dependence on Substrate Temperature

- Optimization of Doping Level

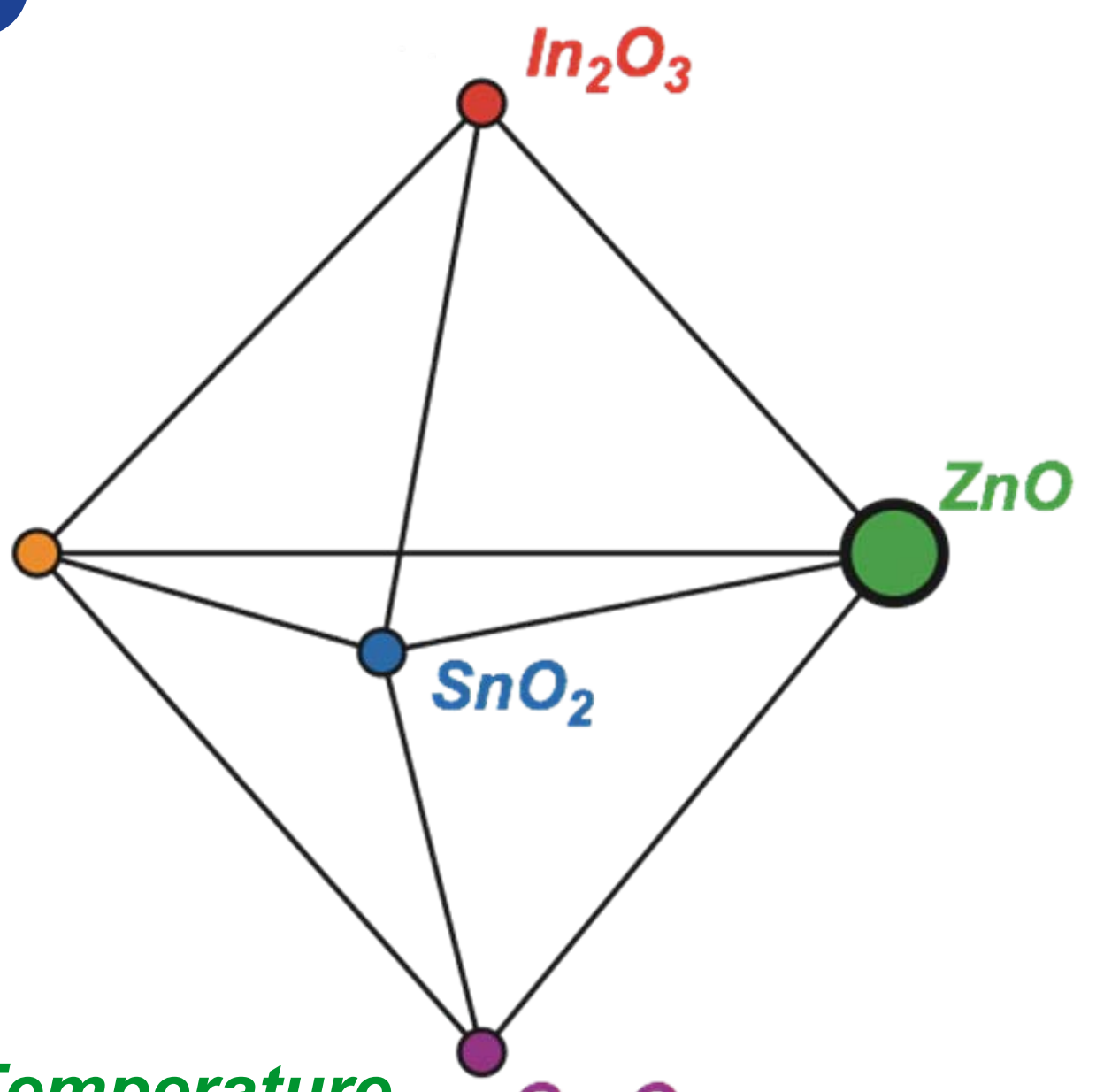

$\mathrm{Ga}_{2} \mathrm{O}_{3}$ 


\section{Combinatorial Tools}

Chemical

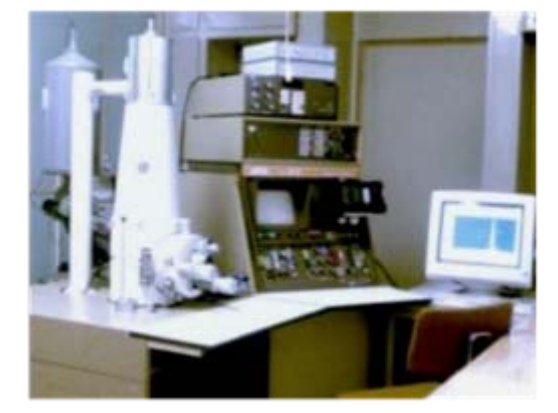

Optical

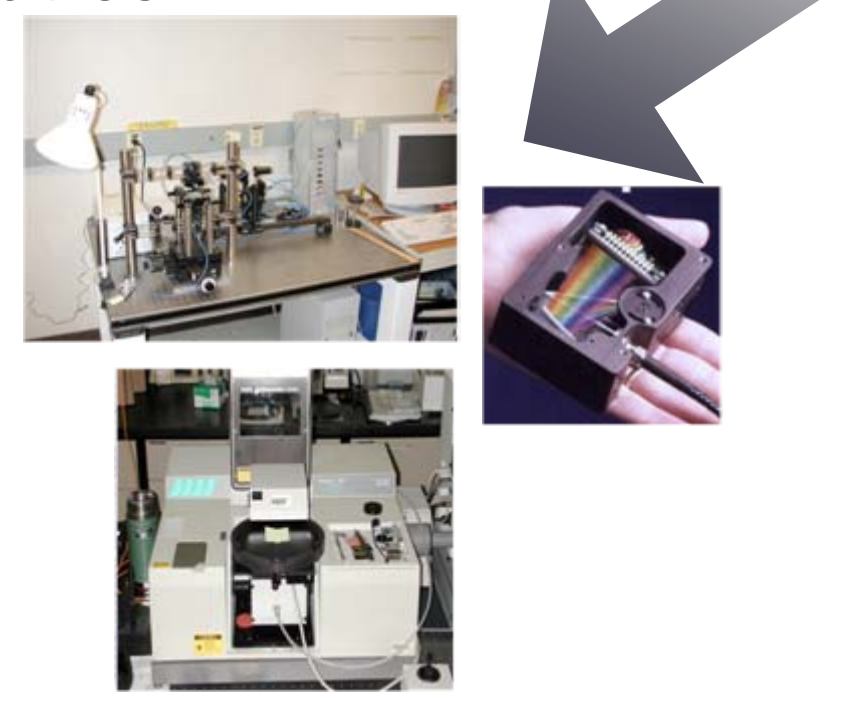

Combi Deposition (graded sputtering)
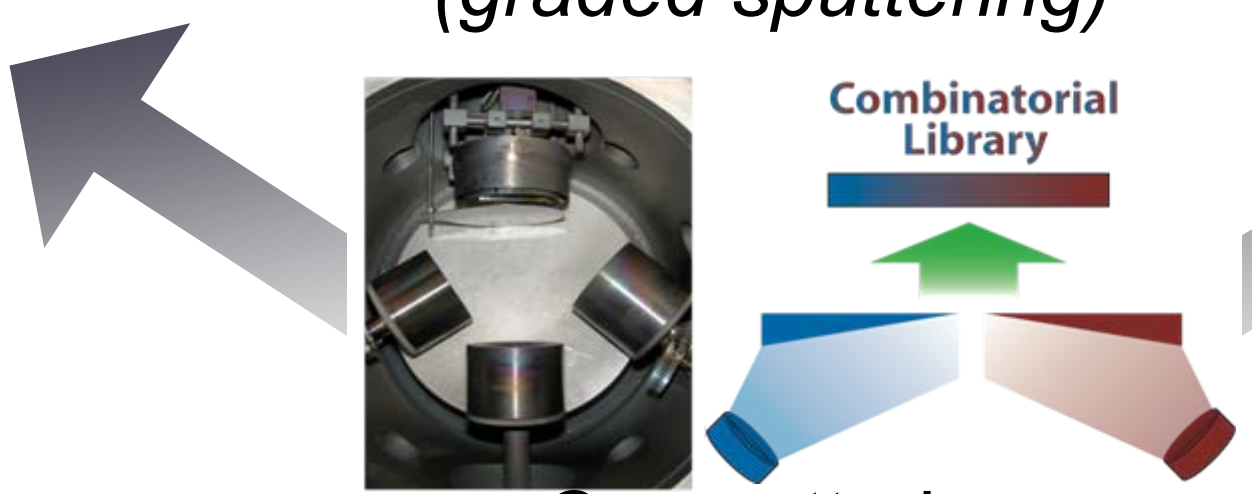

\section{Co-sputtering}

$5 \mathrm{~cm} \times 5 \mathrm{~cm}$ glass substrates

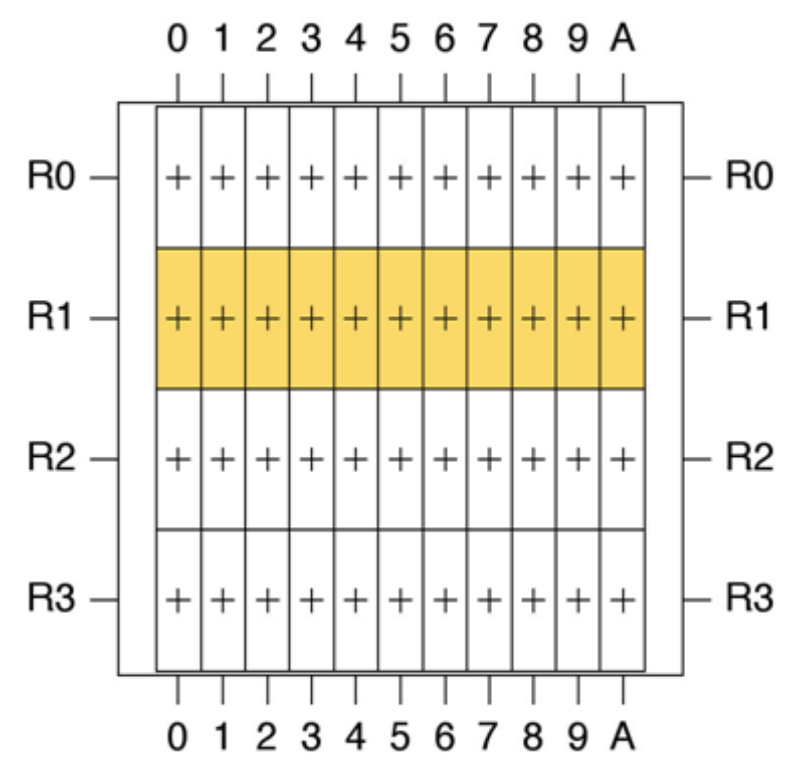

Electrical

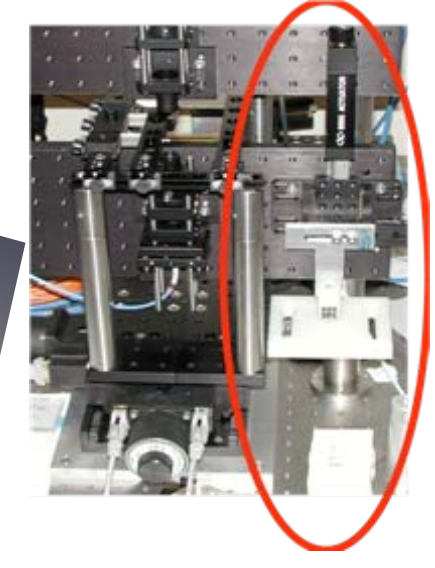

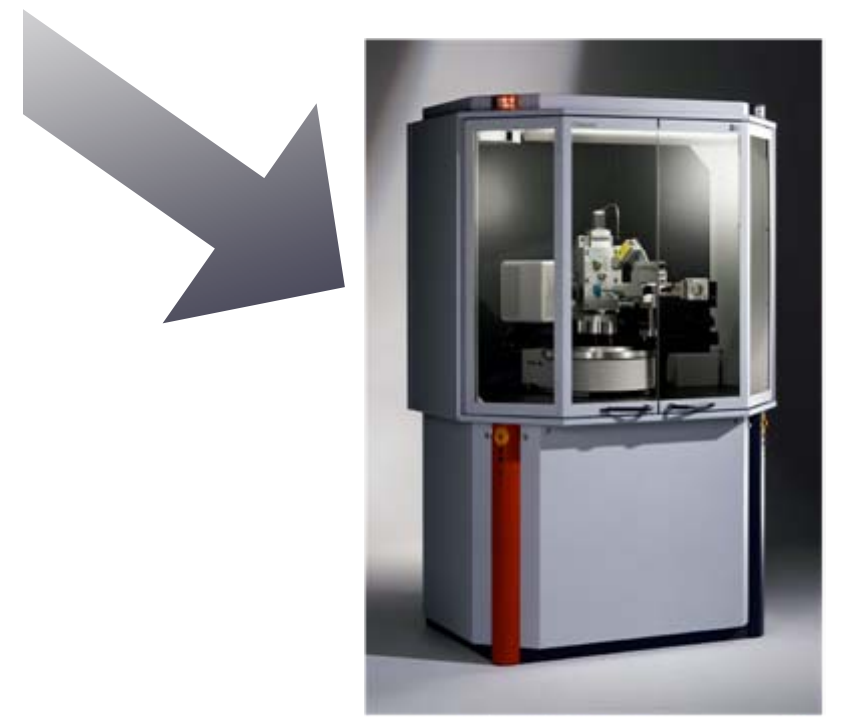

Structural 


\section{Pulsed Laser Deposition}

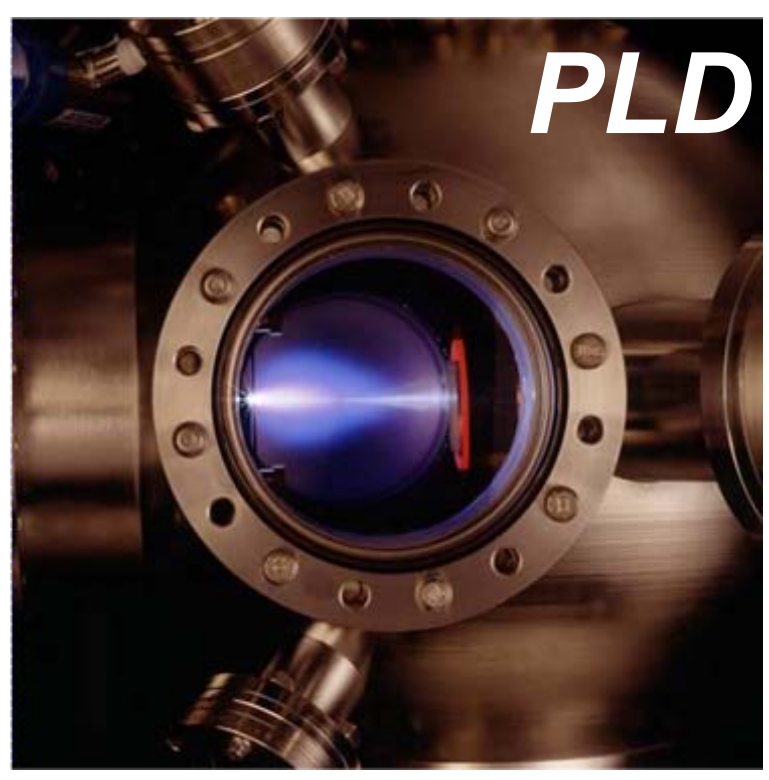

-Substrates:

-Glass (Corning Eagle 2000)

-Deposition:

UV Laser

-Nominal Substrate Temperature:

, 28-300 ${ }^{\circ} \mathrm{C}$

-Targets:

- Ga:ZnO (Plasma Materials)

-Ga composition 2, 5, 8 at.\% (nominal)

Vacuum Chamber

NREL is operated by Midwest Research Institute - Battelle 


\section{Ga:ZnO Structural Properties}

Ga:ZnO deposited at $T_{\text {sub }}=28^{\circ} \mathrm{C}$



Ga:ZnO deposited at $\mathrm{T}_{\text {sub }}=300{ }^{\circ} \mathrm{C}$

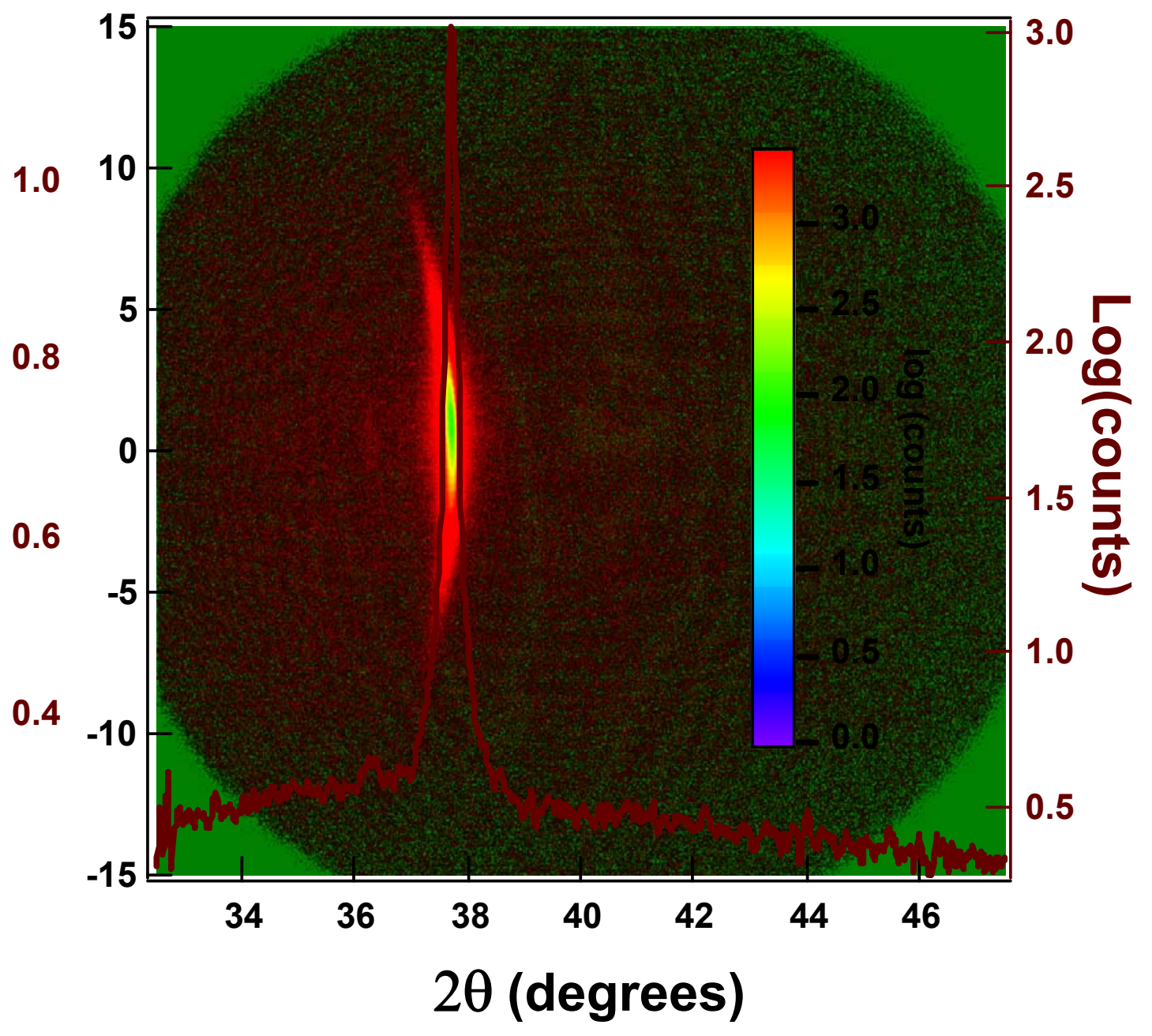


NPEL National Renewable Energy Laboratory

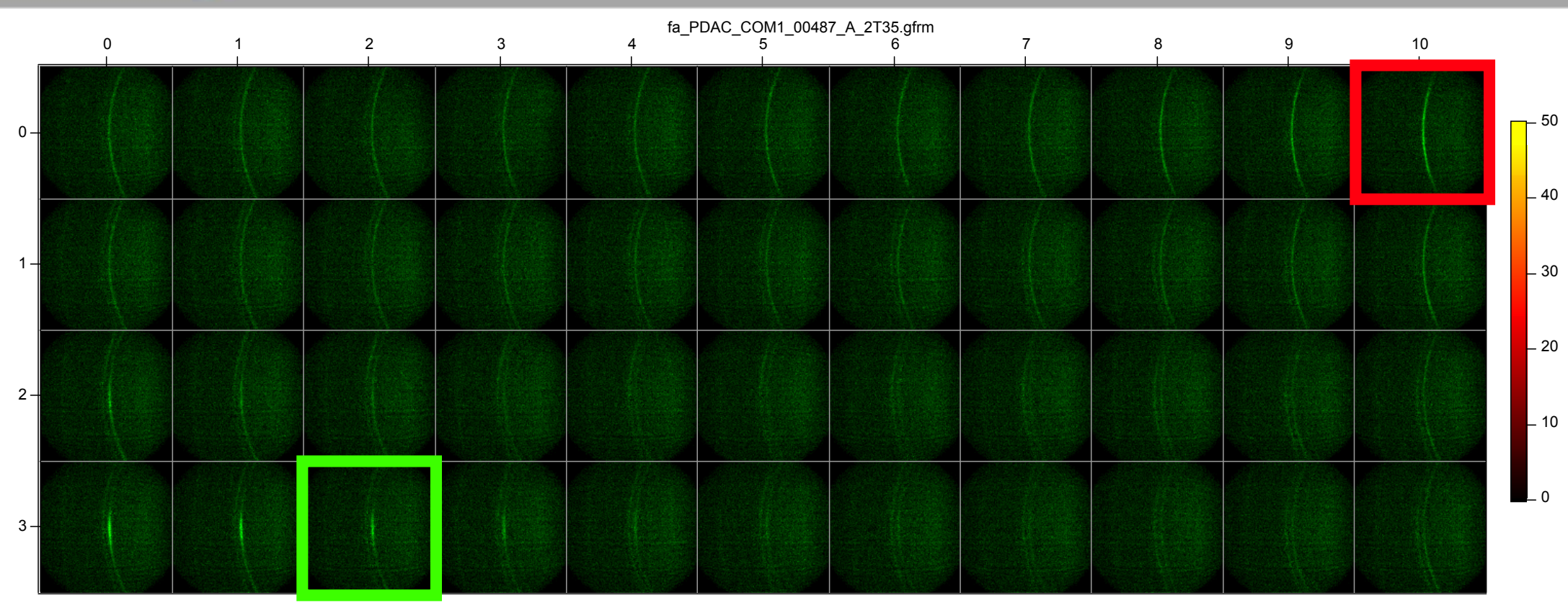

\section{PDAC_COM1_00487_A_2T35_035_000}

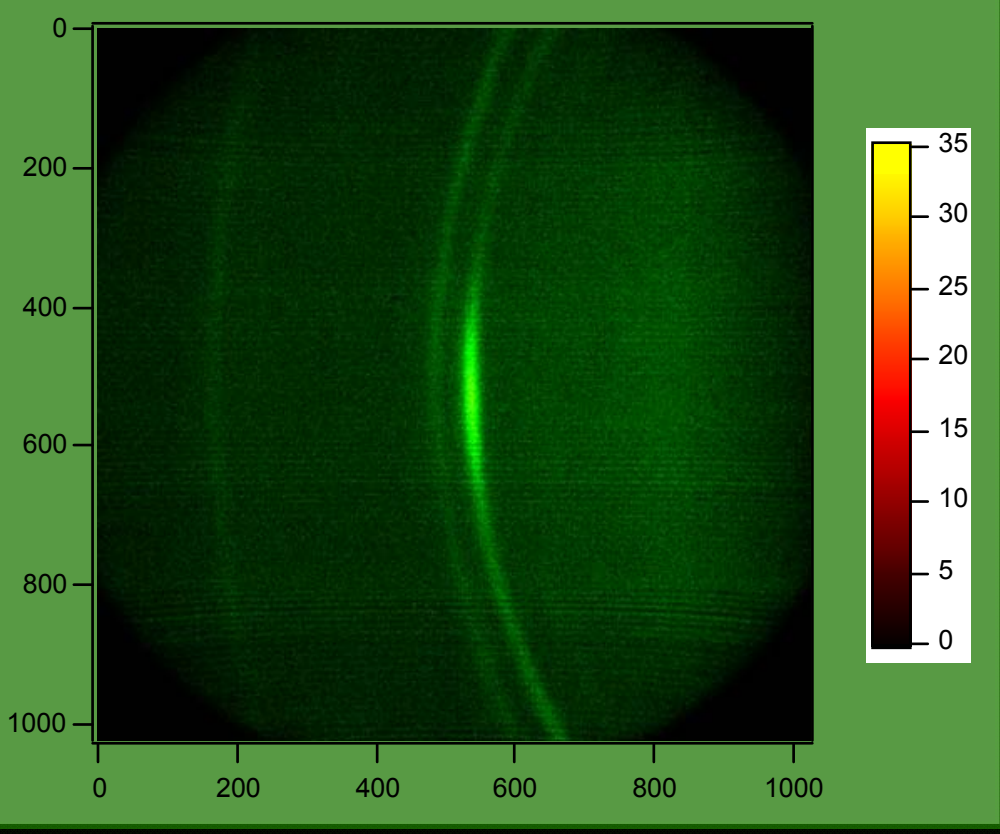

01234456789 A

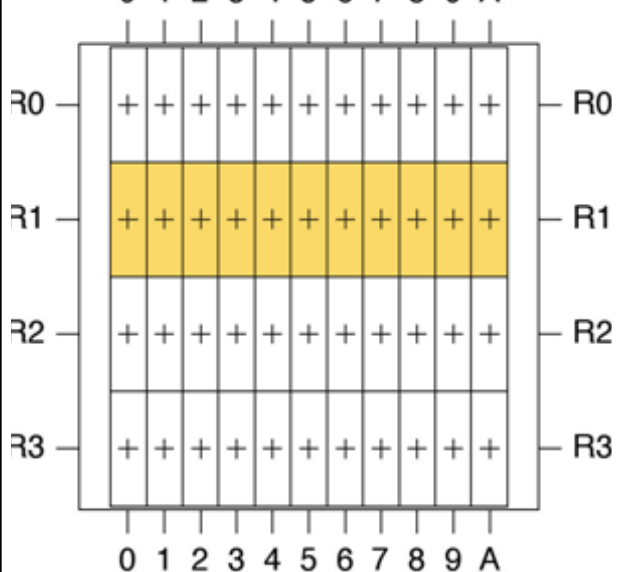

01233456789 A

\section{PDAC_COM1_00487_A_2T35_011_000}

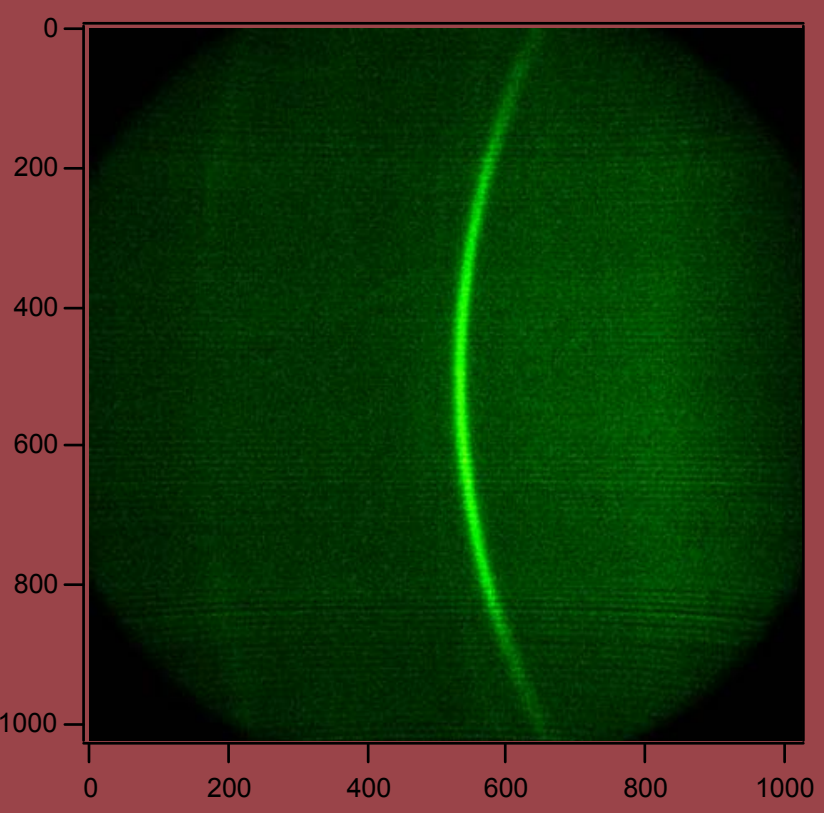




\section{XRD Data: Combinatorial Samples}

Ga:ZnO deposited at $\mathrm{T}_{\text {sub }}=300{ }^{\circ} \mathrm{C}$

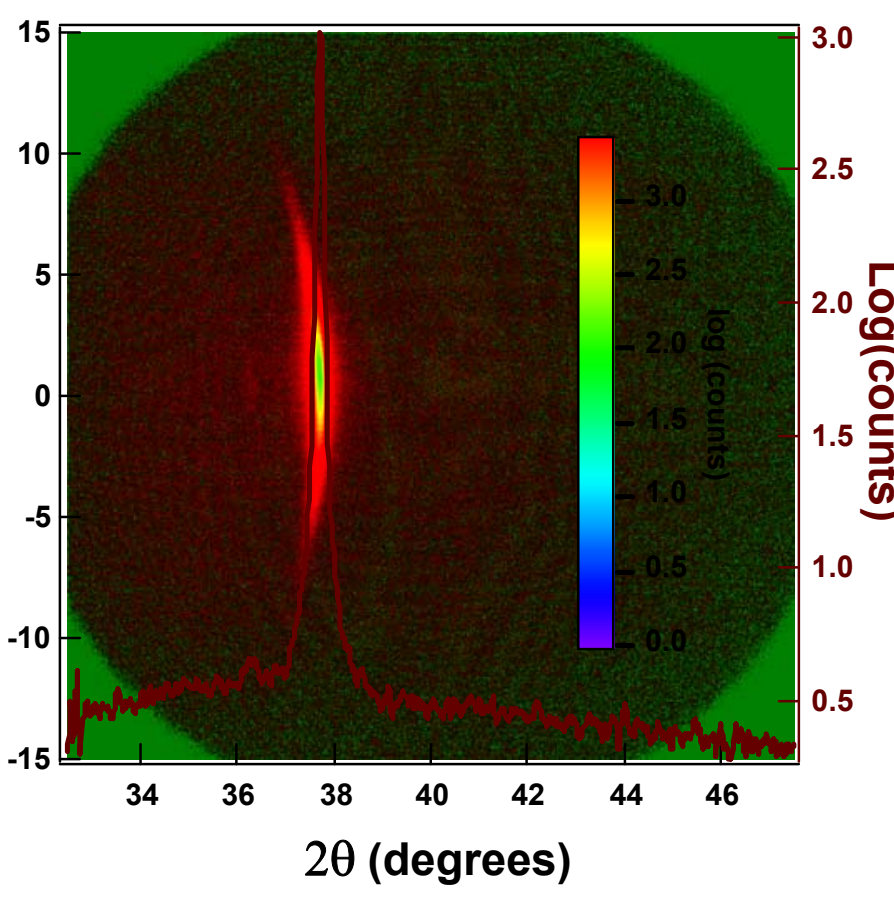

01223456789 A

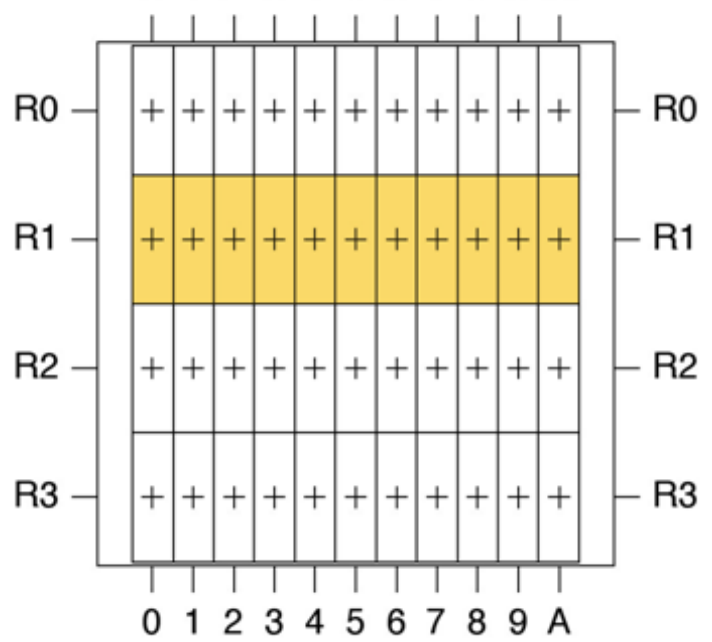

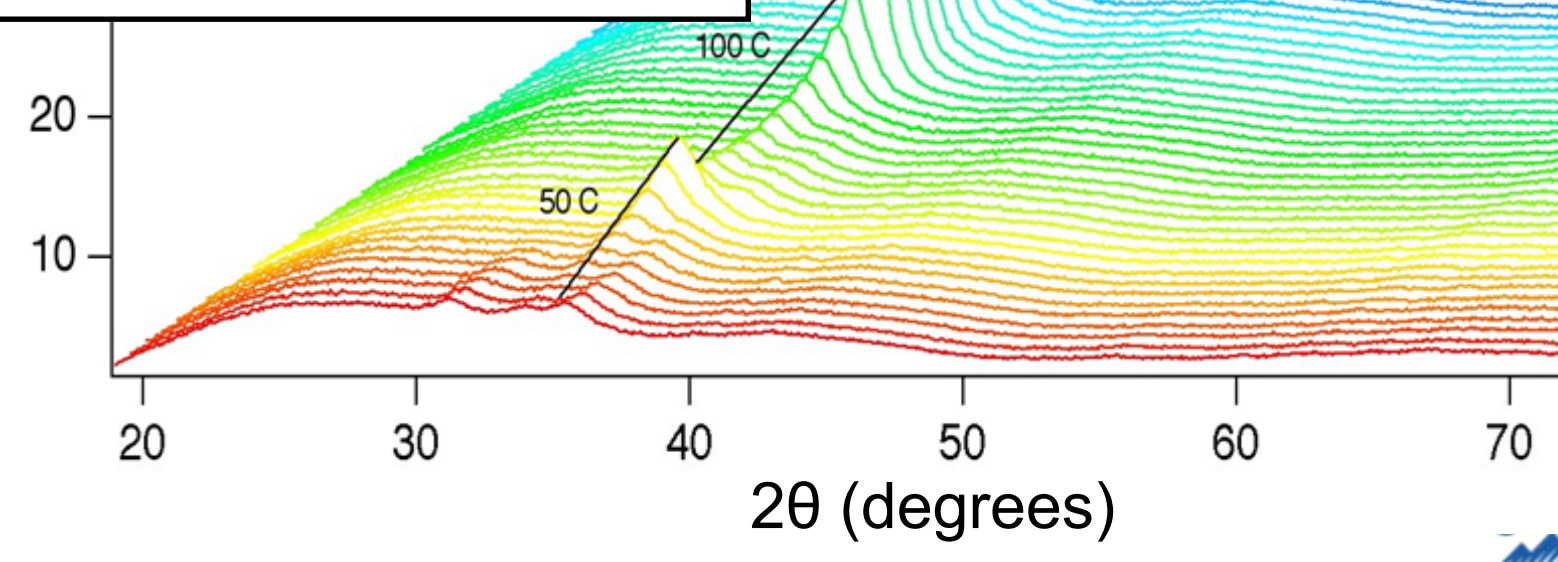

NREL is operated by Midwest Research Institute - Battelle 


\section{Combinatorial GZO}

$x$ position $(\mathrm{mm})$
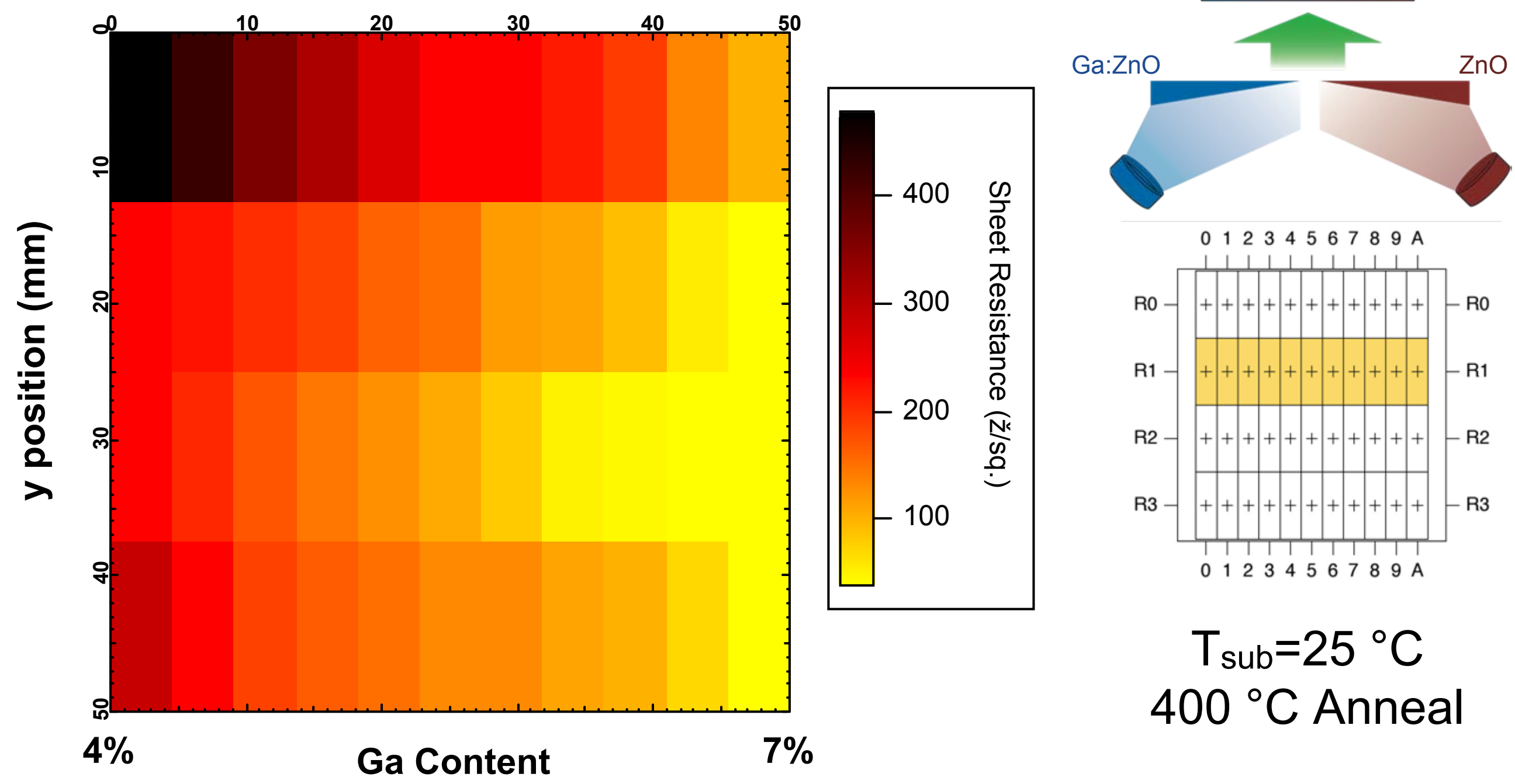

$\begin{array}{lllllllllll}0 & 1 & 2 & 3 & 4 & 5 & 6 & 7 & 8 & 9 & \mathrm{~A}\end{array}$

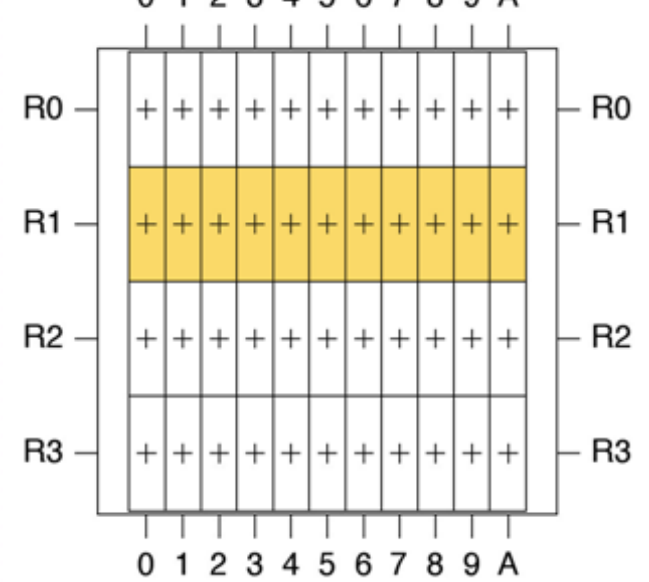

$\mathrm{T}_{\text {sub }}=25{ }^{\circ} \mathrm{C}$ $400{ }^{\circ} \mathrm{C}$ Anneal 


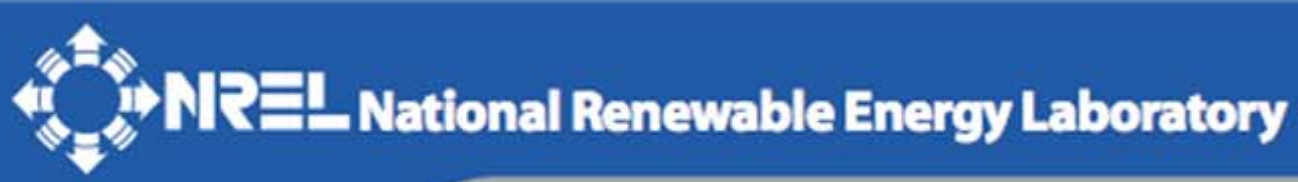

\section{Combinatorial GZO}
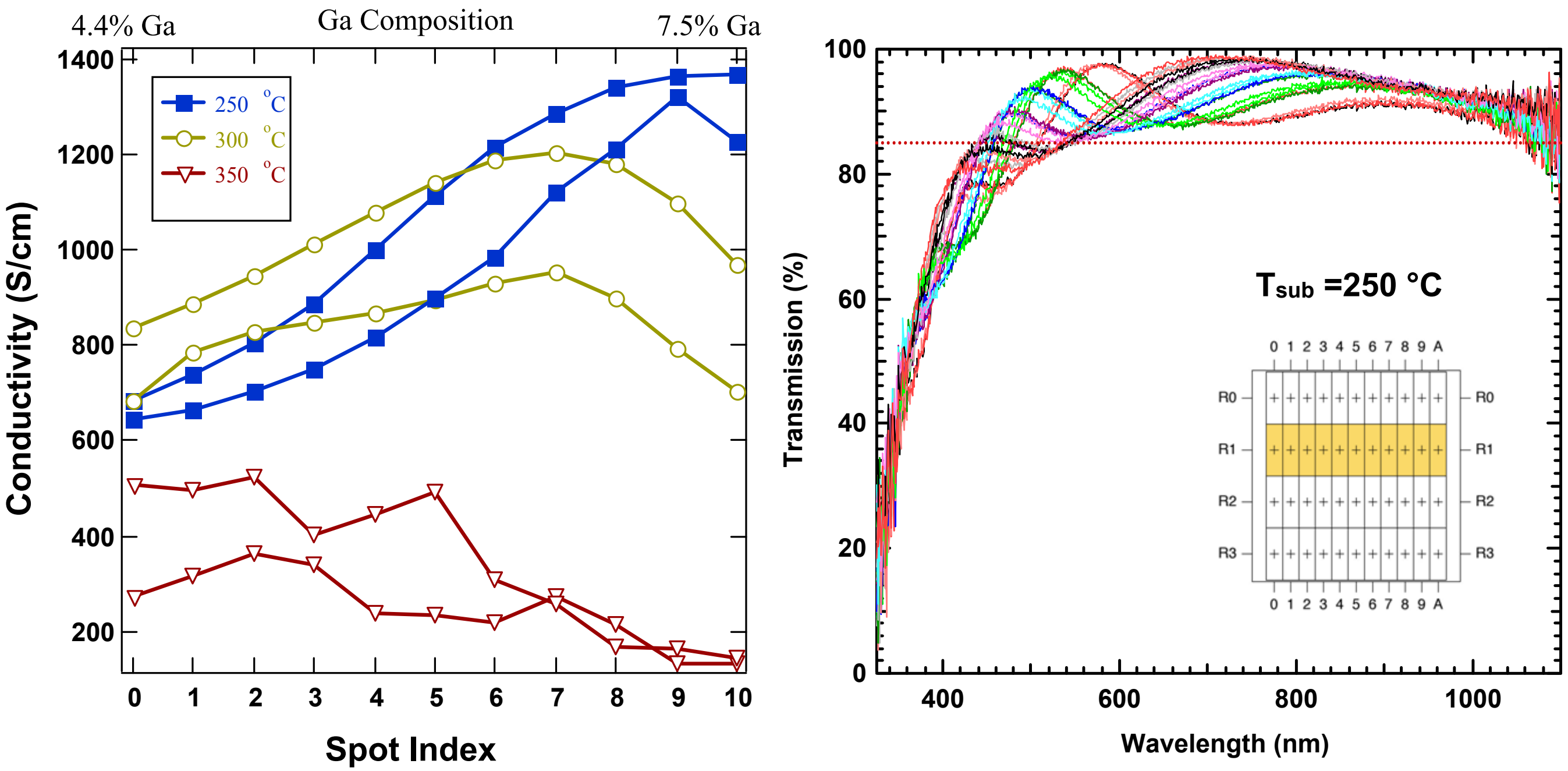

Conductivity across center of several Ga:ZnO compositional libraries 


\section{PLD Ga:ZnO Transport Properties}

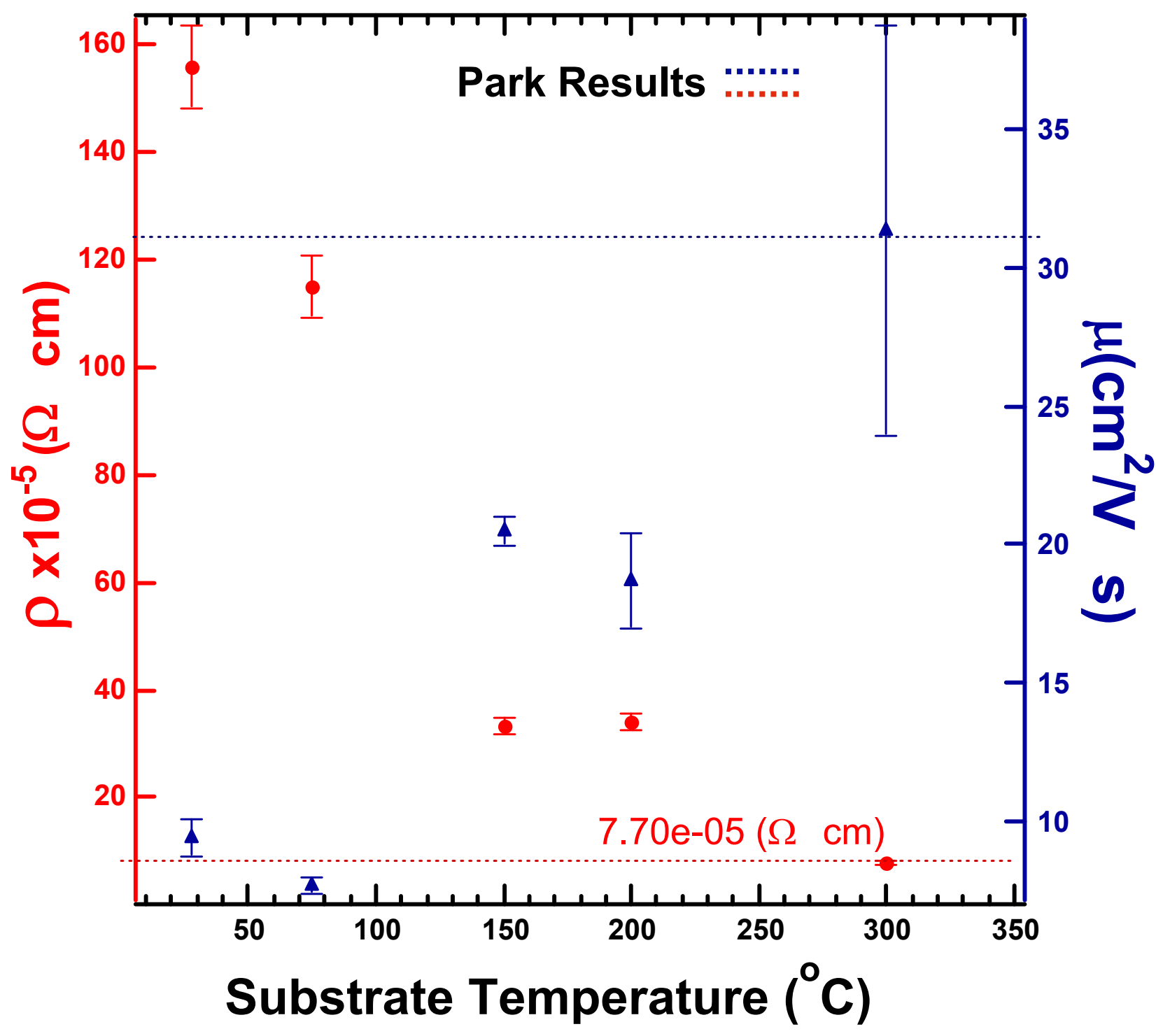

\section{Park Results}

[Thin Solid Films, 513:90-94, 2006]

$$
\begin{array}{r}
\rho=8.12 \times 10^{-5}(\Omega \cdot \mathrm{cm}) \\
\mu=30.96\left(\mathrm{~cm}^{2} V^{-1} s^{-1}\right)
\end{array}
$$

$\sum_{n}^{\frac{E}{3}}$

$$
\begin{gathered}
\text { NREL/PNNL } \\
\rho=7.7 \pm 0.5 \times 10^{-5}(\Omega \cdot \mathrm{cm}) \\
\mu=31 \pm 7\left(\mathrm{~cm}^{2} V^{-1} \mathrm{~s}^{-1}\right)
\end{gathered}
$$

-Base Pressure: $<1.0 \times 10^{-6}$ torr -Deposition Atmosphere: 1.1 mtorr $\mathrm{O}_{2}$ - $\mathrm{KrF}$ Laser ( $\lambda=\mathbf{2 4 8} \mathbf{~ n m})$

-Power Density: $0.76 \mathrm{~J} / \mathrm{cm}^{2}$ 


\section{Sputtered GZO vs. PLD}

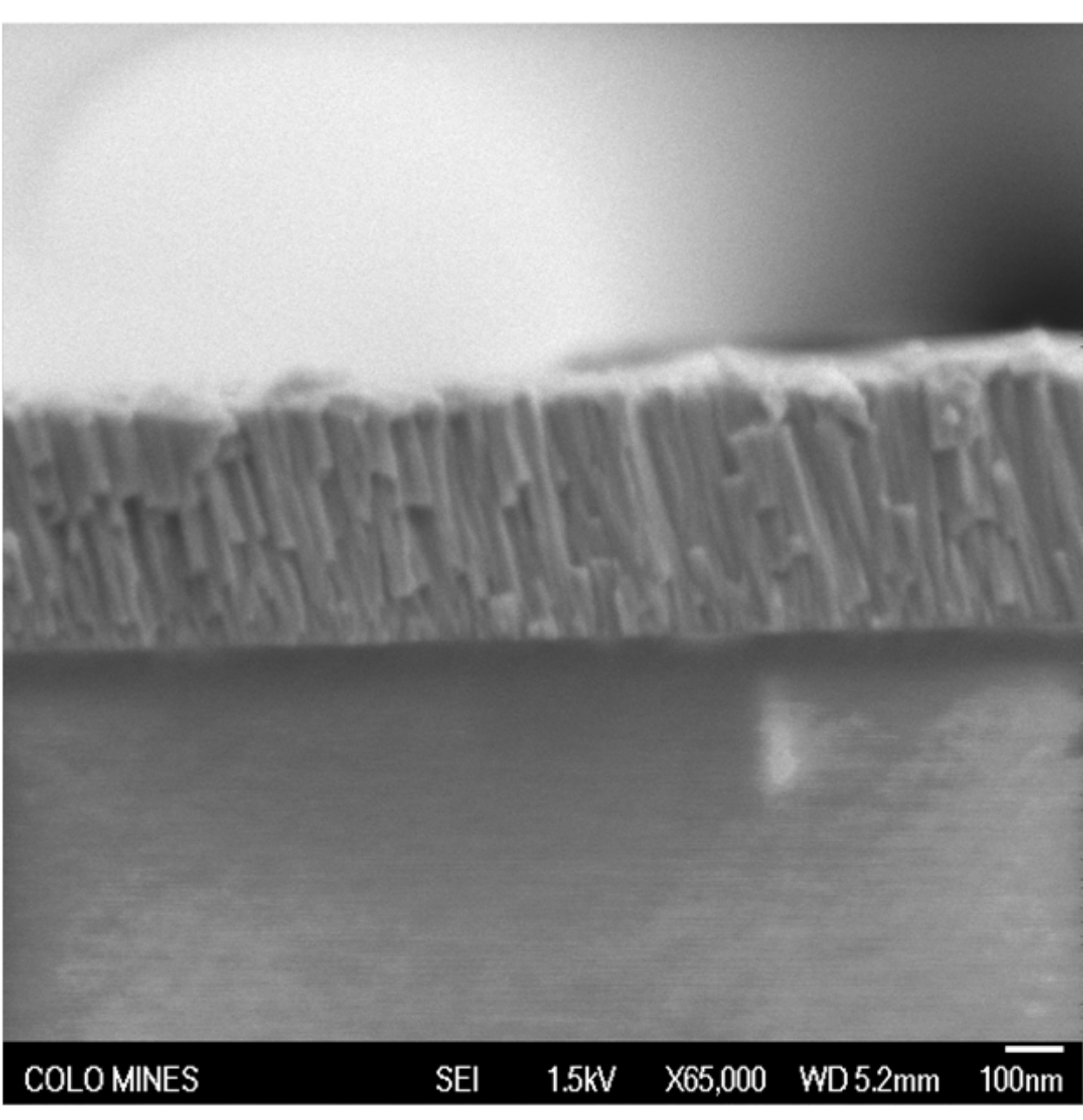

Sputtered Sample

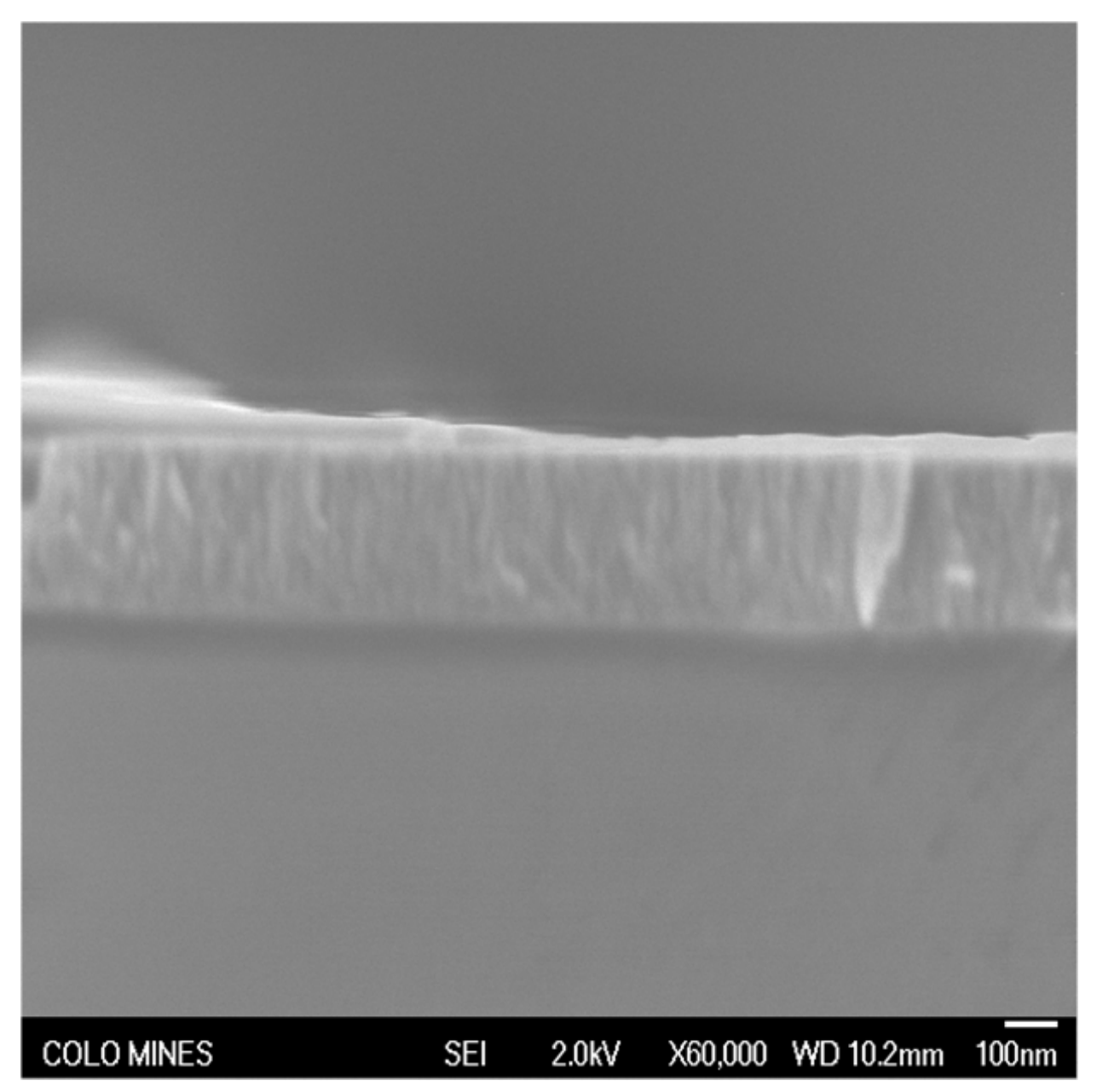

PLD Sample 


\section{Promise \& Future Directions}

-Ga:ZnO from PLD • Expand Combinatorial Studies

, $\sigma_{G Z O} \equiv \sigma_{I T O}$

-Greater Range of Ga a.t.\%

-Scalability?

-Increased Temperature Range $\left(550^{\circ} \mathrm{C}\right)$

-Sputtered Material

-Tuned MgGaZnO for PV applications

$. \sigma_{G Z O}<\sigma_{I T O}$

-PLD

-More Optimization

-Digital Alloys

, $(\sigma, \mu, c . c$.

-Interface Engineering 


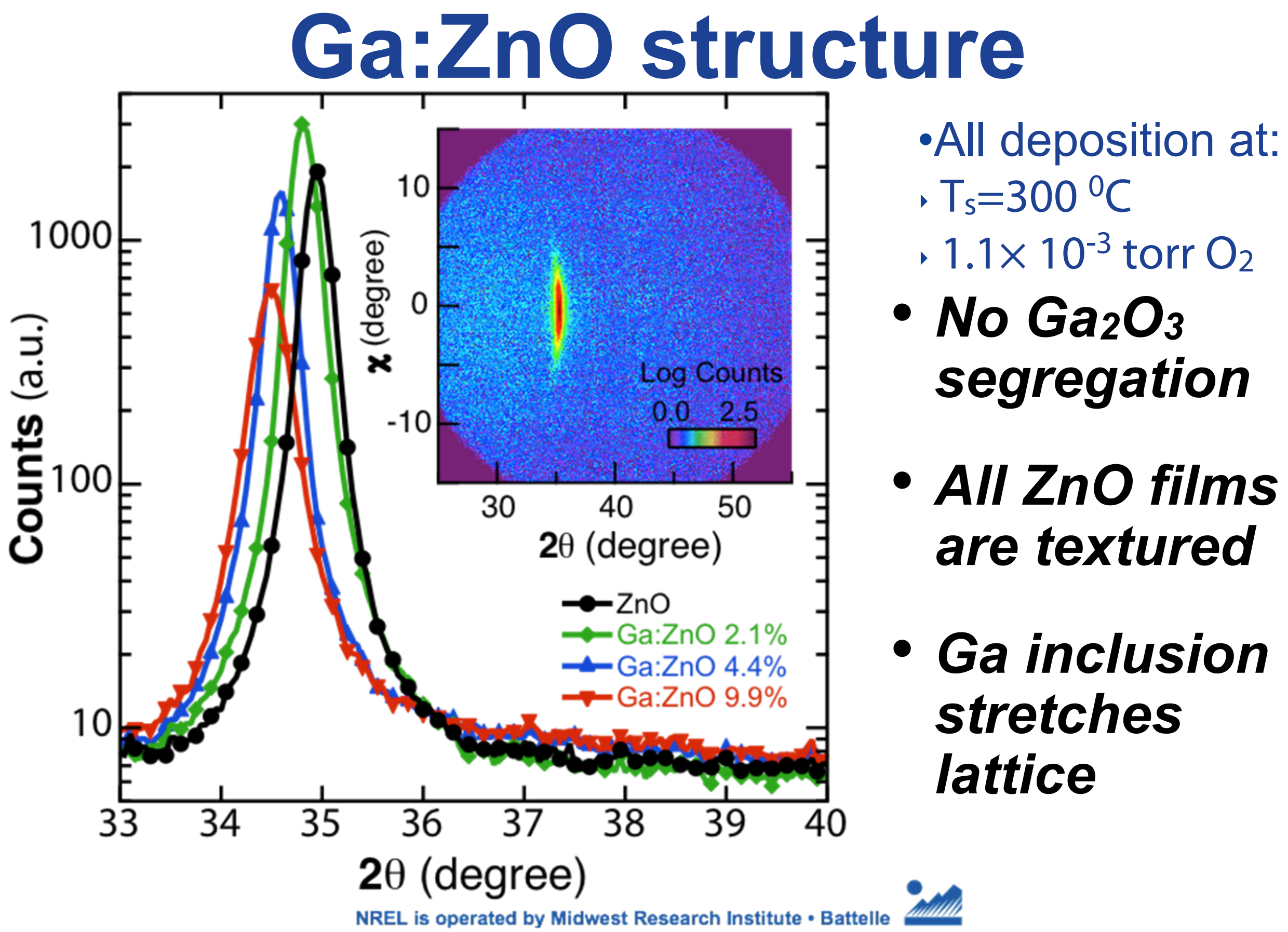

